\title{
FFH-BFSK Multiuser Detection in Uncoordinated Narrow-band FH Systems
}

\author{
Yi-Chen Chen \\ Institute of Communication Engineering \\ College of Electrical Engineering and Computer Science \\ National Taiwan University \\ Taipei, Taiwan 10617. \\ Email: stellar@santos.ee.ntu.edu.tw
}

\author{
Kwang-Cheng Chen \\ Institute of Communication Engineering \\ College of Electrical Engineering and Computer Science \\ National Taiwan University \\ Taipei, Taiwan 10617. \\ Email: chenkc@cc.ee.ntu.edu.tw
}

\begin{abstract}
We propose a multiuser detection scheme for channelized fast frequency hopping with binary frequency shift keying in uncoordinated, narrow-band frequency hopping systems. "Multiuser" here means uncoordinated users and the desired user. This scheme consists of channel state detector and multiuser detector. Both detectors are Gaussian approximated energy combiners and are sub-optimal in maximum a posteriori probability sense. In a symbol interval, the channel state detector first detects the existence of narrow-band frequency hopping signals for each chip, and then the multiuser detector properly combines received chip energies to make the decision. Simulation results show the superiority of this scheme over other existing approaches.
\end{abstract}

\section{INTRODUCTION}

Due to today's fast growing demand for data transmission, the information is transmitted by several collocated and uncoordinated sources. In frequency hopping $(\mathrm{FH})$ multiple access systems, the multiuser interference due to interfering from narrow-band FH signals within other collocated and uncoordinated systems does exist and remains a threat to the desired system. The corresponding multiuser detector to counteract narrow-band, unequal power, uncoordinated $\mathrm{FH}$ signals, however, has not been investigated yet.

[1] proposed two-user multiuser detector, which can afford more simultaneous users. The constraint is that the two-user detector relies a priori design of hopping patterns of all active users and the detection is based on the known received signal amplitude in AWGN channel. However, these requirements are hard to achieve in the uncoordinated FH system and fading environment.

[2]-[4] proposed multilevel FSK multistage detectors based on co-channel interference (CCI) cancellation. The CCI cancellation also needs the knowledge of active users' hopping patterns, which are usually unavailable in uncoordinated FH interfering environment. Thus the detectors in this case are not suitable. Furthermore, their FHMA/MFSK structure requires received energies at all possible bands and has large expense in realization.

This paper proposes a scheme to deal with uncoordinated FH signals with narrower bandwidth and unequal power compared to the desired fast frequency hopping (FFH) signal. We adopt channelized [1] FH-BFSK system which has cheaper implementation. Note that a priori design of hopping patterns [1]-[4] for the uncoordinated interfering users is not possible in the consideration of this paper. Here we focus on two random hopping manner types of the uncoordinated FH signals: pseudo-independent type and independent type. We do not need to know the received envelopes of active users required in [1].

The detection of signal can be viewed as hypothesis testing problem. The channel state (i.e. the existence and the number of the narrow-band signals colliding with the desired signal) dominates the whole performance. However, due to the hopping manner, it has distribution and is not always constant under each hypothesis. The resultant composite hypothesis testing is often quite involved. It is clear that whatever test we design can never be better than a hypothetical test in which we are able to first acquire the channel state information, and then design the optimum likelihood ratio test [6]. Doing this also eliminates some parameters in the composite hypothesis so as to avoid complex calculation.

Specifically, the proposed scheme consists of channel state detector (CSD) and multiuser detector (MD). The CSD utilizes the fact that narrow-band FH signal dwells at a band longer than the desired FFH signal, to first detects the channel state for each FFH chip. After knowing this information, the MD then properly combines received chip energies to make the final decision. Both CSD and MD are sub-optimized by Gaussian approximation to reduce the complexity. We compare the proposed scheme with FH multilevel FSK detectors [2], twouser multiuser detectors [1], equal-gain and self-normalized diversity combining receivers. We also demonstrate the case that the CSD is skipped, to show the effectiveness of the CSD. Furthermore, the maximum-likelihood (ML) scheme is adopted in case of unknown number of the uncoordinated narrow-band signals. This ML scheme is invulnerable to the unknown number and remains superior to other existing approaches.

\section{SySTEM MOdEL}

We adopt the channelized [1] non-coherent FFH-BFSK system, in which two FFH chips are hopped per symbol. That is, $T_{b}=2 T_{c}$, where $T_{b}$ is symbol rate and $T_{c}$ is chip rate. 
The entire spread spectrum band, $W_{s s}$, is partitioned into $N_{t}$ tones, where $N_{t} \leq W_{s s} T_{c}$. These tones are equally spaced and further partitioned into non-overlapping FFH bands ${ }^{1}$. Let $S$ be the power of the transmitted signal for the desired user. The chip energy $E_{c}=S T_{c}$. The channel is single path Rayleigh fading given by $h(t)=\beta \delta(t) \exp (j \theta)$, where $\beta$ is the amplitude of the path and follows Rayleigh distribution. It is assumed that $\beta$ and $\theta$ are statistically independent. The average power of the single path amplitude, $\overline{\beta_{0}^{2}}$, equals 1 .

Timing recovery is assumed to be ideally done at the receiver. The desired signal received at the receiver of the desired user is

$$
S(t)=\sum_{m \geq 0} \beta \sqrt{2 S} p\left(t-m T_{c}\right) \cos \left(2 \pi\left(f_{c}(m)+f_{i}(m)\right) t+\theta\right),
$$

where $p(t)$ is the normalized rectangular pulse function with interval $T_{c} . f_{c}(m)$ is the carrier frequency during the $m$-th chip interval and is controlled by the hopping pattern of the desired user. $f_{0}(m)=-1 /\left(2 T_{c}\right)$ corresponds to the symbol 0 and $f_{1}(m)=1 /\left(2 T_{c}\right)$ corresponds to the symbol 1 . Both symbols are equally probable. Let $b_{n}$ be the desired user's $n$ th transmitted symbol, where $n=1,2, \cdots$. The first chip of $b_{n}$ is hopped in the $(m=2 n-2)$-th chip interval and the second chip is hopped in the $(m=2 n-1)$-th chip interval. We assume $f_{c}(2 n-2)$ and $f_{c}(2 n-1)$ are different.

Consider there are $J$ narrow-band FH users from another uncoordinated system sharing $W_{s s}$ with the desired user. We assume each of the $J$ FH users adopts non-coherent FH-BFSK modulation with signal power $\gamma S$, and symbol rate $1 / T$, where $T=2 T_{c}$ (i.e. twice the desired user's chip interval). In order to simplify the analysis, it is assumed that there is one hop per symbol for the uncoordinated FH signals, and one hopping interval of the uncoordinated FH signals is synchronized to one symbol interval of the desired user. The uncoordinated FH signal at the receiver of the desired user is $I(t)=$

$$
\sum_{j=1}^{J} \sum_{m \geq 0} \beta \sqrt{2 \gamma S} p(t-m T) \cos \left(2 \pi\left(f_{o}^{j}(m) \pm \frac{1}{2 T}\right) t+\phi^{j}(m)+\theta\right) \text {. }
$$

Fig. 1 shows the relationship between the desired user's carriers and narrow-band user's carriers. Note that

$f_{o}^{j}(m) \in\left\{\cdots, f_{c}(m)-\frac{3}{2 T_{c}}, f_{c}(m)-\frac{1}{2 T_{c}}, f_{c}(m)+\frac{1}{2 T_{c}}, \cdots\right\}, \forall j, m$.

We consider two hopping manners for these $J$ users: (1) pseudo-independent type: In each hopping interval, the $J$ FH signals randomly dwell at $J$ distinct $\mathrm{SFH}^{2}$ bands (i.e. there is at most one FH interfering signal dwelling at one SFH band). (2) independent type: In each hopping interval, the $J$ FH signals hop independently (i.e. there can be two or more interfering FH signals dwelling at one SFH band). The received signal is $R(t)=S(t)+I(t)+n(t)$, where $n(t)$ is AWGN with two-sided power spectral density $N_{0} / 2$. Let

\footnotetext{
1"FFH band" denotes a band used by the desired FFH user (e.g. C in Fig. 1)

2"SFH band" denotes a band used by uncoordinated users (e.g. C1 or C2 in Fig. 1).
}



Fig. 1. FFH signal interfered by narrow-band FH signals

$\bar{y}_{m}=\left[\begin{array}{llll}y_{m, c 0} & y_{m, s 0} & y_{m, c 1} & y_{m, s 1}\end{array}\right]$ be the received vector for the $m$-th chip of the desired user. $y_{m, c i}$ and $y_{m, s i}$ are respectively in-phase and quadrature components defined by

$$
\begin{aligned}
& y_{m, c i}=\int_{m T_{c}}^{(m+1) T_{c}} R(t) \sqrt{2 / T_{c}} \cos \left(2 \pi\left(f_{c}(m)+f_{i}(m)\right) t\right) d t \\
& y_{m, s i}=\int_{m T_{c}}^{(m+1) T_{c}} R(t) \sqrt{2 / T_{c}} \sin \left(2 \pi\left(f_{c}(m)+f_{i}(m)\right) t\right) d t .
\end{aligned}
$$

The received vector for the first chip of $b_{n}$ is $\bar{y}_{2 n-2}$ and that for the second chip is $\bar{y}_{2 n-1}$.

\section{Channel State Detection}

At each of $f_{c}(2 n-2)$ and $f_{c}(2 n-1)$, rather than receive the signal only in the corresponding chip interval, the desired user now keeps receiving at the chip frequency in the other chip interval, to get the channel state of that chip frequency. Doing this utilizes the fact that narrow-band FH signal's dwelling interval is longer than the chip interval of the desired user. The desired user can observe the interval in which only the interfering FH signals are present or not, to detect more reliably whether the chip frequency is dwelled by narrow-band FH users.

Specifically, let $\bar{x}_{m}=\left[\begin{array}{llll}x_{m, c 0} & x_{m, s 0} & x_{m, c 1} & x_{m, s 1}\end{array}\right]$ be the received channel state vector for the $m$-th chip of the desired user. For $m=2 n-2$ (i.e. the first chip of $b_{n}$ ), we have

$$
\begin{aligned}
& x_{m, c i}=\int_{(m+1) T_{c}}^{(m+2) T_{c}} R(t) \sqrt{2 / T_{c}} \cos \left(2 \pi\left(f_{c}(m)+f_{i}(m)\right) t\right) d t, \\
& x_{m, s i}=\int_{(m+1) T_{c}}^{(m+2) T_{c}} R(t) \sqrt{2 / T_{c}} \sin \left(2 \pi\left(f_{c}(m)+f_{i}(m)\right) t\right) d t,
\end{aligned}
$$

For $m=2 n-1$ (i.e. the second chip of $b_{n}$ ), we have

$$
\begin{aligned}
& x_{m, c i}=\int_{(m-1) T_{c}}^{m T_{c}} R(t) \sqrt{2 / T_{c}} \cos \left(2 \pi\left(f_{c}(m)+f_{i}(m)\right) t\right) d t, \\
& x_{m, s i}=\int_{(m-1) T_{c}}^{m T_{c}} R(t) \sqrt{2 / T_{c}} \sin \left(2 \pi\left(f_{c}(m)+f_{i}(m)\right) t\right) d t,
\end{aligned}
$$




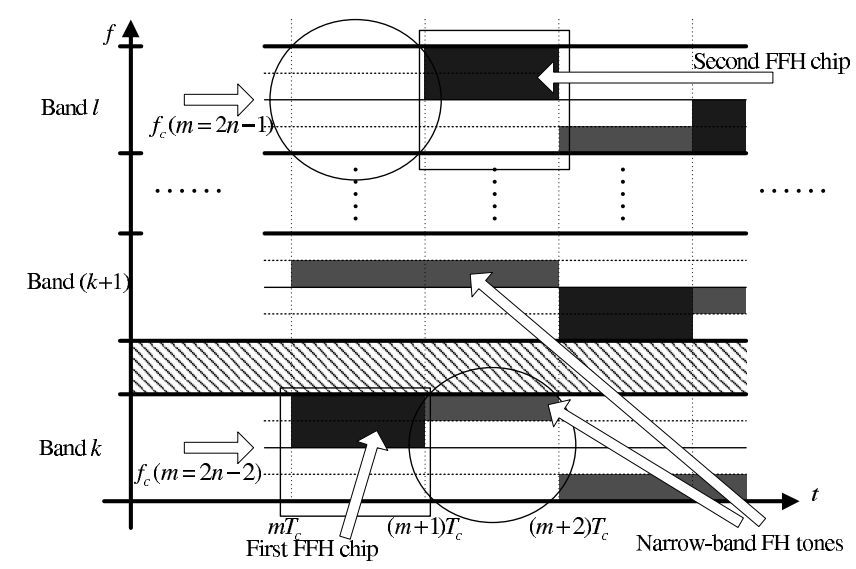

Fig. 2. Demodulation intervals and channel state observation intervals in the proposed scheme

Fig. 2 shows the differences between demodulation intervals (marked with rectangles) of $\bar{y}_{m}$, and channel state observation intervals of $\bar{x}_{m}$ (marked with circles). Note that during the same symbol period, $\bar{y}_{m}$ is received in a given chip interval while $\bar{x}_{m}$ is always received in the other chip interval.

Among $J$ uncoordinated users, we assume $r$ users transmit tones in $\mathrm{C} 11, d-r$ users transmit tones in $\mathrm{C} 12, s$ users transmit tones in $\mathrm{C} 21$ and $e-s$ users transmit tones in $\mathrm{C} 22$, as indicated in Fig. 1. Pair $\{d, e\}$ is the channel state to detect. Given $d$ and $e, E(r, s)$ denotes the event described in Fig. 1. It happens with probability $p(E(r, s) \mid\{d, e\})=\left(\begin{array}{l}d \\ r\end{array}\right)\left(\begin{array}{l}e \\ s\end{array}\right)(1 / 2)^{d+e}$.

It can be shown that for the pseudo-independent $\mathrm{FH}$ type, $p(\{d, e\})=\left(\begin{array}{c}2 N_{c}-2 \\ J-d-e\end{array}\right) /\left(\begin{array}{c}2 N_{c} \\ J\end{array}\right)$ and $d, e \in\{0,1\}$.

For independent type narrow-band FH, $d, e \in\{0,1, \cdots, J\}$. We have $p(\{d, e\})=\left(\begin{array}{l}J \\ d\end{array}\right)\left(\begin{array}{l}J-d \\ e\end{array}\right)\left(1 / 2 N_{c}\right)^{(d+e)}\left(1-1 / N_{c}\right)^{(J-d-e)}$.

Based on Bayes' formula, the a posteriori probability for $\{d, e\}$ can be written as:

$\left.p\left(\{d, e\} \mid \bar{x}_{m}\right)=\sum_{r=0}^{d} \sum_{s=0}^{e} p(\{d, e\}, E(r, s)) \mid \bar{x}_{m}\right)$.

We can then derive the decision rule by maximizing a posteriori probability (MAP) for $\{d, e\}$ given $\bar{x}_{m}:\{\hat{d}(m), \hat{e}(m)\}$ $=\arg \max p\left(\{d, e\} \mid \bar{x}_{m}\right)=\arg \max W_{1}\left(\bar{x}_{m} \mid\{d, e\}\right)$, $\{d, e\}$ where $W_{1}\left(\bar{x}_{m} \mid\{d, e\}\right)=$

$$
p(\{d, e\})\left\{\sum_{r=0}^{d} \sum_{s=0}^{e} p\left(\bar{x}_{m} \mid\{d, e\}, E(r, s)\right) p(E(r, s) \mid\{d, e\})\right\} .
$$

The expression of $p\left(\bar{x}_{m} \mid\{d, e\}, E(r, s)\right)$ is in APPENDIX I. For the $m$-th chip, the CSD picks $\{\hat{d}(m), \hat{e}(m)\}$ such that $W_{1}\left(\bar{x}_{m} \mid\{\hat{d}(m), \hat{e}(m)\}\right) \geq W_{1}\left(\bar{x}_{m} \mid\{d, e\}\right), \forall d, e$.

The CSD is calculated by Gaussian approximation [5] and is sub-optimal in MAP sense. It performs multiple composite hypothesis test for $\{\hat{d}(m), \hat{e}(m)\}$ (hypothesis parameter is now reduced to $\{r, s\}$ pair only). Based on (9), Fig. 3 depicts the decision metric of $\{d, e\}$ in the CSD. The total number of components summed in (9) depends on $\{d, e\}$ pair. Given $\{d, e\}$, one can further prove this number is at most four for pseudo-independent type narrow-band $\mathrm{FH}$ signals, and at most $(\lfloor J / 2\rfloor+1)(\lceil J / 2\rceil+1)$ for independent type narrow-band $\mathrm{FH}$, where $\lfloor x\rfloor$ denotes integer part of $x$, and $\lceil x\rceil=\lfloor x\rfloor+1$.

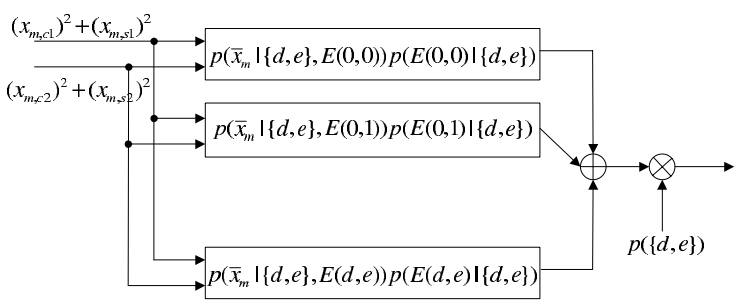

Fig. 3. Decision metric of $\{d, e\}$ in the CSD

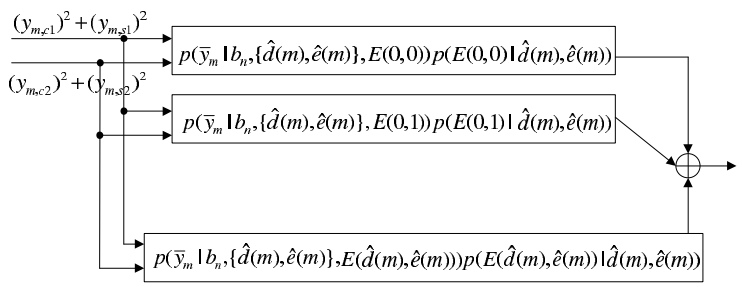

Fig. 4. Decision metric of $b_{n}$ for the $m$-th chip in the MD

\section{Multiuser Detection}

After getting $\{\hat{d}(m), \hat{e}(m)\}$, we proceed to detect $b_{n}$. It is assumed that the two received chips are uncorrelated and the MD picks $\hat{b}_{n}$ with the maximum $\prod_{m=2 n-2}^{2 n-1} W_{2}\left(\bar{y}_{m} \mid \hat{b}_{n},\{\hat{d}(m), \hat{e}(m)\}\right)$, where

$W_{2}\left(\bar{y}_{m} \mid \hat{b}_{n},\{\hat{d}(m), \hat{e}(m)\}\right)=\sum_{r=0}^{\hat{d}(m)} \sum_{s=0}^{\hat{e}(m)}$

$p\left(\bar{y}_{m} \mid b_{n},\{\hat{d}(m), \hat{e}(m)\}, E(r, s)\right) p(E(r, s) \mid\{\hat{d}(m), \hat{e}(m)\})$.

The expression of $p\left(\bar{y}_{m} \mid b_{n},\{\hat{d}(m), \hat{e}(m)\}, E(r, s)\right)$ is in APPENDIX II. Like the CSD, the MD is a Gaussian approximated sub-optimal non-linear energy combiner. It performs binary composite hypothesis test for $\hat{b_{n}}$. Based on (10), Fig. 4 depicts the decision metric of $b_{n}$ for the $m$-th chip in the MD. Given $\hat{d}(m)$ and $\hat{e}(m)$, the total number of components summed in $(10)$ is $(\hat{d}(m)+1)(\hat{e}(m)+1)$.

Fig. 5 depicts the system block diagram of the proposed scheme. We can further see that at the receiver two energy detectors are required in order to simultaneously get $\bar{y}_{m}$ and $\bar{x}_{m+1}$ (with $m=2 n-2$ ), or $\bar{y}_{m}$ and $\bar{x}_{m-1}$ (with $m=2 n-1$ ). One is for $f_{c}(2 n-2)$ and the other is for $f_{c}(2 n-1)$.

\section{Simulation Results}

We adopt the data rate $32 \mathrm{kbps}$ and the total bandwidth greater than $8.192 \mathrm{MHz}$ as a representative example of the medium number of hopping bands for general use. We set $N_{c} 128$ in this case. Furthermore, we set $E_{b} / N_{0}=40 \mathrm{~dB}$ and $\gamma=3$. Repetition code (odd diversity $L=9$ ) and hard decision with majority vote are adopted. Fig. 6 and Fig. 7 show the capacities in terms of coded bit error rate versus number of uncoordinated FH users. The coded bit error rate is calculated by $\sum_{l=(L+1) / 2}^{L}\left(\begin{array}{l}L \\ l\end{array}\right)\left(P_{b}\right)^{l}\left(1-P_{b}\right)^{L-l}$, where $P_{b}$ is the un-coded simulated bit error rate.

For multilevel FSK detectors [2], we adopt two hops per bit (number of hops is 14 for every 7 bits). $M$ equals $N_{c}$, where $M$ is the symbol alphabet size defined in [2]. Fig. 6(a) and Fig. 7 (a) show that at bit error rate $10^{-4}$, the proposed 


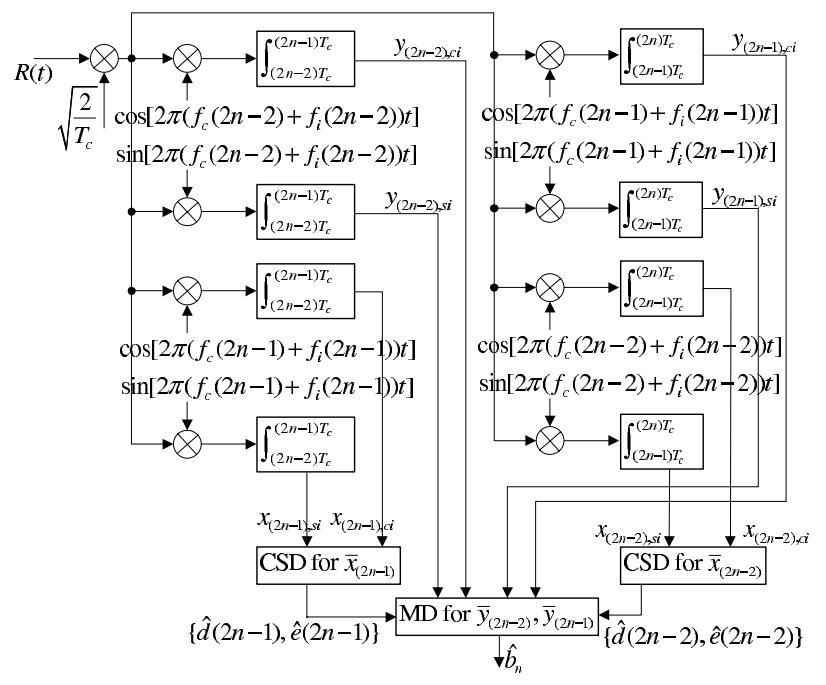

Fig. 5. Block diagram of the proposed scheme

scheme can support around 15 users more than the multilevel FSK detector does.

For two-user detectors [1], we denote "side information" the knowledge of received envelope and whether the desired user uses the common band with uncoordinated users in each hop. In Fig. 6(a) and Fig. 7(a), we can see that the twouser detector is not suitable, because its assumption [1] that "any user is hit at most by one user" does not apply to this uncoordinated situation. In addition, the two-user detector requires the additional knowledge of received envelope and hopping patterns of interfering users.

TABLE I summarizes the testing formulations of the proposed scheme in four cases. In case the total user number $J$ is unknown, a priori probability of $\{d, e\}$ hypothesis is not available and it is assumed uniform for every possible $\{d, e\}$. We call the proposed scheme adopted in this case the maximum likelihood (ML) scheme. It is also assumed that there is at most one FH interfering user dwelling at one SFH band $^{3}$. The ML scheme is compared to the EG receiver and SN receiver in Fig. 6(b) and Fig. 7(b). At bit error rate 10 $10^{-4}$, it supports around 10 users more than $\mathrm{SN}$ receiver, and 40 users more than the EG receiver in both types of narrowband FH signals. By further comparing the MAP scheme and ML scheme in Fig. 6(a) and 6(b), or in Fig. 7(a) and $7(b)$, we can see that there is slight difference in performance curves. Therefore the proposed scheme is invulnerable to the unavailability of total user number $J$.

In addition, we demonstrate the effectiveness of CSD. In Fig 6(a) or 6(b), we compare the scheme with CSD and the scheme without CSD. Comparing these two curves we can see that there is performance degradation of the scheme without CSD, though it is still superior to other methods.

\footnotetext{
${ }^{3}$ Though this assumption does not satisfy the independent type, we use it since the number of hypotheses is unavailable in this case. In fact, this number is $(J+1)(J+2) / 2$, which also results in huge complexity for large $J$. This assumption results in another sub-optimal solution while with much less complexity.
}

TABLE I

TEsting Formulations in 4 CASES

\begin{tabular}{|c|l|l|}
\hline & $J$ is known & $J$ is unknown \\
\hline MD & Case 1: & Case 3: \\
only & MD performs binary & MD performs binary \\
& composite hypothesis test for & composite hypothesis test for \\
& $\hat{b}_{n}$ (hypothesis parameters: & $\hat{b}_{n}$ (hypothesis parameters: \\
& $\{d, e\}$ and $\{r, s\}$ ) and is sub- & $\{d, e\}$ and $\{r, s\}$ ) and is sub- \\
& optimal in MAP sense & optimal in ML sense \\
\hline CSD & Case 2: & Case 4: \\
and & CSD performs multiple & CSD performs multiple \\
MD & composite hypothesis test for & composite hypothesis test for \\
& $\{d, e\}$ (hypothesis parameters: & $\{d, e\}$ (hypothesis parameters: \\
& $\{r, s\}$ ) and is sub-optimal in & $\{r, s\}$ ) and is sub-optimal in \\
& MAP sense. Given $\{\hat{d}, \hat{e}\}$, & ML sense. Given $\{\hat{d}, \hat{e}\}$, \\
& MD performs binary compo- & MD performs binary compo- \\
& site hypothesis test for $\hat{b}_{n}$ & site hypothesis test for $\hat{b}_{n}$ \\
& (hypothesis parameters: & (hypothesis parameters: \\
& $\{r, s\}$ ) and is sub-optimal & $\{r, s\}$ ) and is sub-optimal \\
& in MAP sense. & in MAP sense. \\
\hline
\end{tabular}



(a) Capacities of proposed schemes, two-user detectors, and FH multilevel FSK detector.

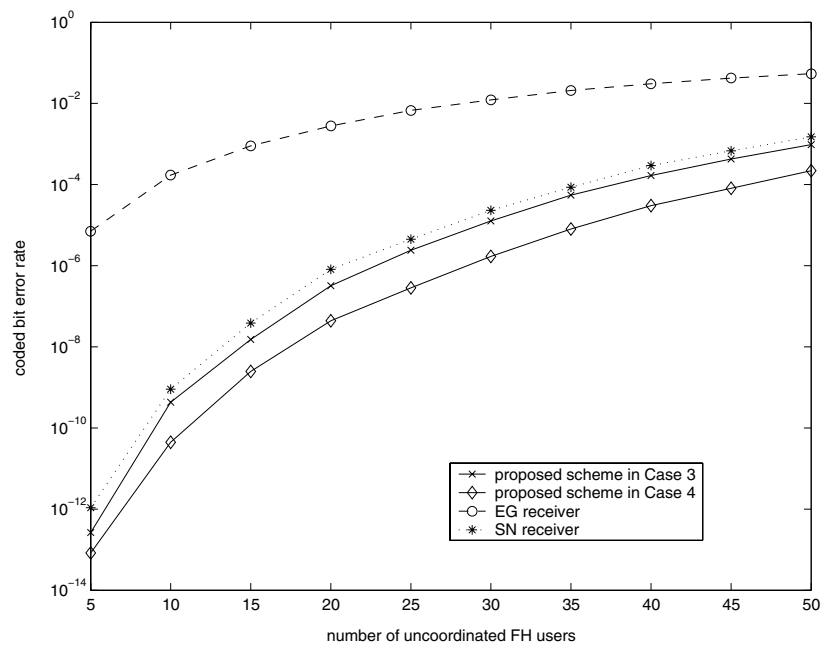

(b) Capacities of proposed schemes, $\mathrm{SN}$ receiver, and EG receiver.

Fig. 6. Capacity comparisons in pseudo-independent narrow-band FH 


\section{CONCLUSION}

We propose a scheme composed of a channel state detector followed by a multiuser detector for channelized FFH-BFSK in uncoordinated narrow-band FH systems. The channel state detector observes the interval in which only the interfering FH signals are present or not (i.e. in the absence of desired FFH signal), to more reliably detect for each chip the existence and the number of narrow-band FH users dwelling in the same band as the desired FFH user does. Based on detected channel state information, multiuser detection is performed on both received chip vectors for each transmitted symbol. Simulation results show the superiority of the proposed scheme over the previous FH multilevel FSK detectors, two-user multiuser detectors, EG and SN receivers. In case of the unknown total number of narrow-band FH users, the ML scheme is adopted without apparent performance degradation.

In summary, combining the channel state detector and the multiuser detector results in more interfering resistance and is more applicable than the existing approaches [1]-[4] for the environment shared by several narrow-band uncoordinated $\mathrm{FH}$ signals.

\section{APPENDIX I \\ EXPRESSION OF $p\left(\bar{x}_{m} \mid\{d, e\}, E(r, s)\right)$}

By Gaussian approximation [5], given $\{d, e\}$ and $E(r, s)$, $x_{m, c i}$ and $x_{m, s i}$ are Gaussian random variables with zero mean and variance $\sigma_{i}^{2}(\{d, e\}, E(r, s))$ in the following.

$\sigma_{1}^{2}(\{d, e\}, E(r, s))=E_{c} \bar{\beta}_{0}^{2} Y_{1} / 2+N_{0} / 2$, $\sigma_{2}^{2}(\{d, e\}, E(r, s))=E_{c} \beta_{0}^{2} Y_{2} / 2+N_{0} / 2$, where

$$
\begin{aligned}
& Y_{1}=(2 \sqrt{2 \gamma} / \pi)^{2}\left(d+s(1 / 3)^{2}+(e-s)(1 / 5)^{2}\right), \\
& Y_{2}=(2 \sqrt{2 \gamma} / \pi)^{2}\left(e+(d-r)(1 / 3)^{2}+r(1 / 5)^{2}\right),
\end{aligned}
$$

It follows that $p\left(\bar{x}_{m} \mid\{d, e\}, E(r, s)\right)=$

$$
\begin{gathered}
\frac{\exp \left(-\left(\left(x_{m, c 1}\right)^{2}+\left(x_{m, s 1}\right)^{2}\right) /\left(2 \sigma_{1}^{2}(\{d, e\}, E(r, s))\right)\right)}{2 \pi \sigma_{1}^{2}(\{d, e\}, E(r, s))} \times \\
\frac{\exp \left(-\left(\left(x_{m, c 2}\right)^{2}+\left(x_{m, s 2}\right)^{2}\right) /\left(2 \sigma_{2}^{2}(\{d, e\}, E(r, s))\right)\right)}{2 \pi \sigma_{2}^{2}(\{d, e\}, E(r, s))} .
\end{gathered}
$$

\section{APPENDIX II \\ EXPRESSION OF $p\left(\bar{y}_{m} \mid b_{n},\{\hat{d}(m), \hat{e}(m)\}, E(r, s)\right)$}

Similarly, it can be shown that $y_{m, c i}$ and $y_{m, s i}$ are Gaussian random variables with zero mean and variance in the following.

$$
\begin{gathered}
\sigma_{1}^{2}\left(b_{n}=1,\{\hat{d}(m), \hat{e}(m)\}, E(r, s)\right)=E_{c} \bar{\beta}_{0}^{2}\left(1+Z_{1}\right) / 2+N_{0} / 2, \\
\sigma_{2}^{2}\left(b_{n}=1,\{\hat{d}(m), \hat{e}(m)\}, E(r, s)\right)=E_{c} \bar{\beta}_{0}^{2} Z_{2} / 2+N_{0} / 2, \\
\sigma_{1}^{2}\left(b_{n}=-1,\{\hat{d}(m), \hat{e}(m)\}, E(r, s)\right)=E_{c} \bar{\beta}_{0}^{2} Z_{1} / 2+N_{0} / 2, \\
\sigma_{2}^{2}\left(b_{n}=-1,\{\hat{d}(m), \hat{e}(m)\}, E(r, s)\right)=E_{c} \bar{\beta}_{0}^{2}\left(1+Z_{2}\right) / 2+N_{0} / 2,
\end{gathered}
$$

where $Z_{i}$ can be obtained by substituting $\hat{d}(m)$ for $d$, and $\hat{e}(m)$ for $e$ in (11) and (12).

We have $p\left(\bar{y}_{m} \mid b_{n},\{\hat{d}(m), \hat{e}(m)\}, E(r, s)\right)$ by substituting $\sigma_{i}^{2}\left(b_{n},\{\hat{d}(m), \hat{e}(m)\}, E(r, s)\right)$ for $\sigma_{i}^{2}(\{d, e\}, E(r, s))$ in (13).

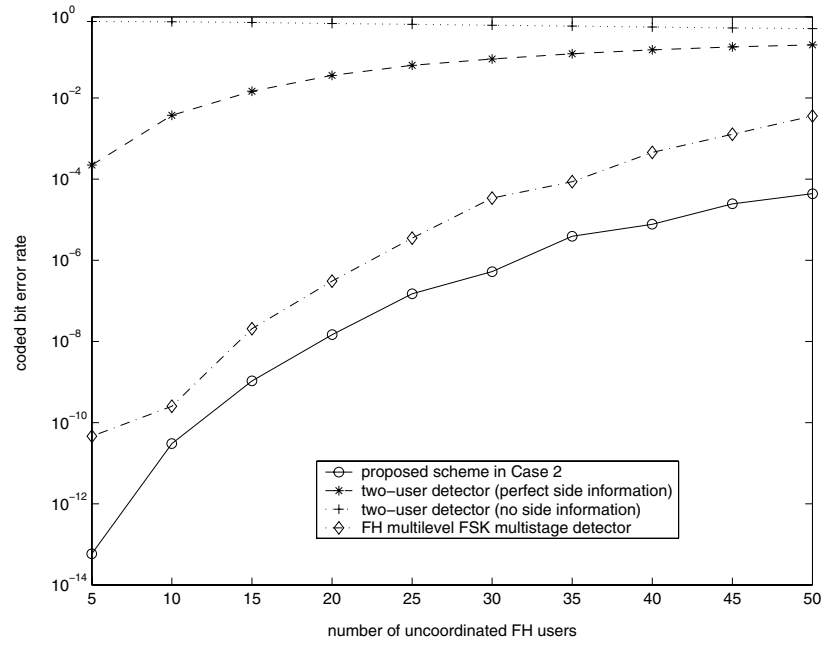

(a) Capacities of proposed schemes, two-user detectors, and FH multilevel FSK detector.

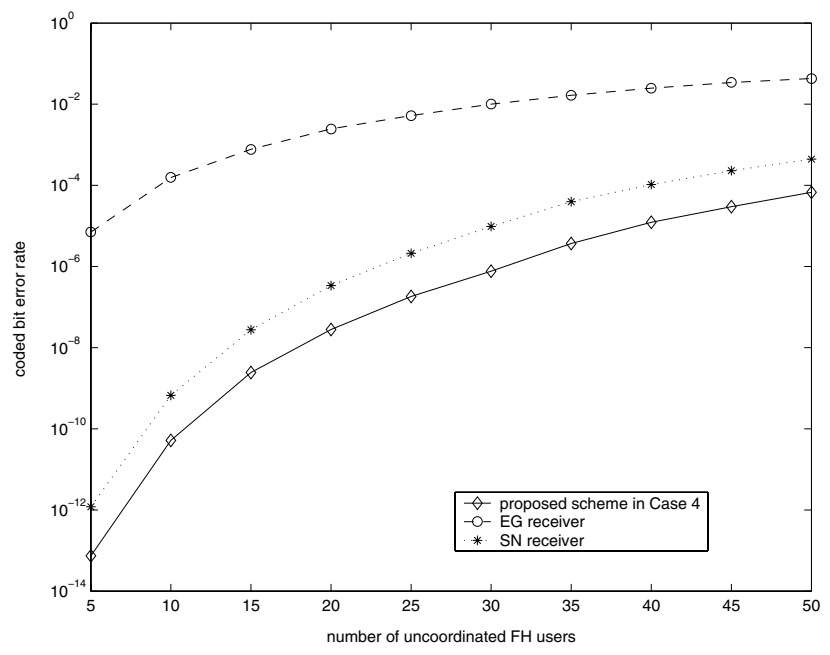

(b) Capacities of proposed schemes, SN receiver, and EG receiver.

Fig. 7. Capacity comparisons in independent narrow-band FH

\section{ACKNOWLEDGMENT}

The authors would like to thank anonymous referees, for their comments and suggestions.

\section{REFERENCES}

[1] T. C. Wu, C. C. Chao and K. C. Chen, "Multiuser Detection for Frequency-Hopped Spread Spectrum Systems with BFSK Modulation,' IEEE, VTC 2001, pp. 323-327, Fall, 2001.

[2] T. Mabuchi, R. Kohno and H. Imai, "Multiuser Detection Scheme Based on Canceling Cochannel Interference for MFSK/FH-SSMA System," IEEE J. Select. Areas Commun., vol. 12, no. 4, pp. 593-604, May 1994.

[3] S. Y. Lin, G. C. Yang, S. C. Tseng and C. F. Hong, "Improved Cochannel Interference Cancellation for MFSK/FH-SSMA Systems," IEEE J. Select. Areas Commun., vol. 17, no. 11, pp. 1940-1952, Nov. 1999.

[4] K. W. Halford, B. P. Maite, "Multistage Multiuser Detection for FHMA," IEEE Trans. Commun., vol. 48, no. 9, pp. 1550-1562, Sep. 2000.

[5] T. C. Wu, C. C. Chao and K. C. Chen, "Capacity of Synchronous Coded DS SFH and FFH Spread-Spectrum Multiple-Access for Wireless Local Communications", IEEE Trans. Commun., vol. 45, no. 2, pp. 200-212, Feb. 1997.

[6] Van Trees, Detection, Estimation, Modulation Theory, 1968. 